\title{
Parabolic Sandwiches for Functions on a Compact Interval and an Application to Projectile Motion
}

\author{
Robert Kantrowitz $\mathbb{D}^{1}$ and Michael M. Neumann $\mathbb{D D}^{2}$ \\ ${ }^{1}$ Mathematics Department, Hamilton College, 198 College Hill Road, Clinton, NY 13323, USA \\ ${ }^{2}$ Department of Mathematics and Statistics, Mississippi State University, Mississippi State, MS 39762, USA \\ Correspondence should be addressed to Robert Kantrowitz; rkantrow@hamilton.edu
}

Received 19 February 2019; Accepted 14 April 2019; Published 2 May 2019

Academic Editor: Brigitte Forster-Heinlein

Copyright (C) 2019 Robert Kantrowitz and Michael M. Neumann. This is an open access article distributed under the Creative Commons Attribution License, which permits unrestricted use, distribution, and reproduction in any medium, provided the original work is properly cited.

\begin{abstract}
About a century ago, the French artillery commandant Charbonnier envisioned an intriguing result on the trajectory of a projectile that is moving under the forces of gravity and air resistance. In 2000, Groetsch discovered a significant gap in Charbonnier's work and provided a valid argument for a certain special case. The goal of the present article is to establish a rigorous new approach to the full result. For this, we develop a theory of those functions which can be sandwiched, in a natural way, by a pair of quadratic polynomials. It turns out that the convexity or concavity of the derivative plays a decisive role in this context.
\end{abstract}

\section{Introduction and Historical Background}

Every differentiable real-valued function $f$ on an interval $[a, b]$ induces a canonical pair of polynomials of degree at most two, namely, the polynomials $p$ and $q$ that coincide with $f$ at the endpoints $a$ and $b$, while their derivatives satisfy $p^{\prime}(a)=f^{\prime}(a)$ and $q^{\prime}(b)=f^{\prime}(b)$. Here we focus on the particular case when $f$ is sandwiched by these polynomials in the sense that either $q \leq f \leq p$ or $p \leq f \leq q$ holds on the entire interval $[a, b]$. The two possibilities are illustrated by the graphics in Figure 1, which are based on certain cubic polynomials $f$.

We will see that, in fact, every cubic polynomial $f$ allows this kind of enveloping on any given interval $[a, b]$, but, in general, the polynomial sandwich condition need not be satisfied even when $f$ is convex or concave on $[a, b]$. For instance, in each of the two cases $f(x)= \pm \sin (x)$ for $0 \leq$ $x \leq \pi$, the corresponding polynomials $p$ and $q$ coincide and hence miserably fail to sandwich $f$.

In the main results of this article, we connect the sandwich property to the convexity or concavity behavior of the derivative of $f$. Specifically, we prove that $q \leq f \leq p$ if $f^{\prime}$ is convex on $[a, b]$ and that $p \leq f \leq q$ if $f^{\prime}$ is concave on $[a, b]$. Moreover, we show that certain localized versions of our sandwich conditions actually characterize the convexity or concavity of $f^{\prime}$. From a broader point of view, while every calculus student knows what the conditions $f^{\prime} \geq 0$ or $f^{\prime \prime} \geq$ 0 on $[a, b]$ mean for a given function $f$, here we offer an interpretation and visualization of the estimate $f^{\prime \prime \prime} \geq 0$ on $[a, b]$ in terms of the parabolic sandwich condition $q \leq f \leq p$ on $[a, b]$ and its localized version.

All this has an interesting history and application. In Chapter V of his treatise on ballistics [1], published in 1907, the French artillery commandant Prosper Charbonnier studied the trajectory traced by a projectile under the forces of gravity and air resistance. Such a trajectory represents, of course, the solution of a certain system of differential equations, but an explicit solution formula is, in general, out of reach. Nevertheless, Charbonnier discovered that the trajectory is always sandwiched by the two parabolas associated with the data at the starting point and a chosen endpoint of the trajectory. These parabolas allow a natural interpretation in terms of projectile motion without air resistance in the classical sense of Galileo and serve to define a certain safety region for the complicated case of air resistance. Groetsch [2] noticed a serious gap in Charbonnier's presentation and provided a rigorous proof but only for the special case of shooting from ground level to ground level.

Although our work is inspired by [2], our methods are rather different and remarkably elementary. The principal 


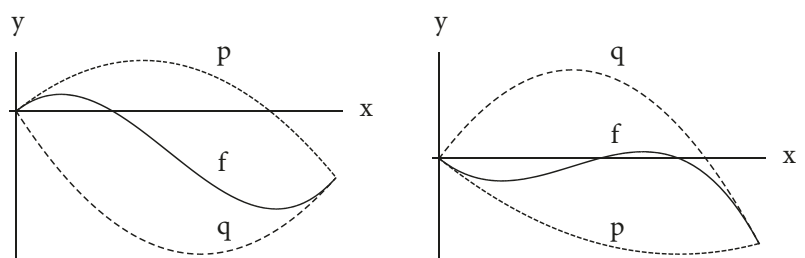

FIGURE 1

tool is a characterization of convex functions in terms of certain average values which is discussed in Section 2. Our sandwich results are then developed in Section 3, while Section 4 provides a simple approach to the full version of what Charbonnier proposed regarding projectile motion under air resistance.

\section{Preliminaries on Convex Functions}

We start by collecting a few tools from the theory of convex functions for which we refer, for instance, to Chapter III of [3] and Chapter I of [4]. A real-valued function $g$ on an interval $J \subseteq \mathbb{R}$ is said to be convex (or concave upward) provided that

$$
g(t u+(1-t) v) \leq \operatorname{tg}(u)+(1-t) g(v)
$$

for all $u, v \in J$ and $t \in(0,1)$. If the preceding inequality holds with " $<$ " instead of " $\leq$ " for all distinct $u, v \in J$, then $g$ is said to be strictly convex. Reversing the inequalities leads to the definitions of concave and strictly concave functions, while affine functions are those that are both convex and concave. It is well known that all such functions are automatically continuous on the interior of $J$. Moreover, a differentiable function is (strictly) convex precisely when its derivative is (strictly) increasing. In particular, if $g$ is twice differentiable on $J$ and satisfies $g^{\prime \prime}(x)>0$ for all $x \in J$, then $g$ is strictly convex. The following simple result will be useful.

Lemma 1. Let $g$ be a convex function on an interval J. If $u, v \in$ $J$ are points with $u<v$ such that the identity

$$
g(t u+(1-t) v)=\operatorname{tg}(u)+(1-t) g(v)
$$

holds for at least one $t \in(0,1)$, then $g$ is actually affine on $[u, v]$. In particular, $g$ is strictly convex precisely when $g$ fails to be affine on each nondegenerate subinterval of $J$.

Proof. Assume that $g$ is not affine on $[u, v]$. By the convexity of $g$, it then follows that there exists some point $x \in(u, v)$ for which $g(x)<\ell(x)$, where $\ell$ denotes the function representing the line through $(u, g(u))$ and $(v, g(v))$. On the other hand, again by convexity, the graph of $g$ on $[u, v]$ is bounded above by the line segments from $(u, g(u))$ to $(x, g(x))$ and from $(x, g(x))$ to $(v, g(v))$. Hence $g(x)<\ell(x)$ entails that $g<\ell$ on $(u, v)$ which means that $g(t u+(1-t) v)<\operatorname{tg}(u)+(1-t) g(v)$ for all $t \in(0,1)$, the desired contradiction. The final claim is now immediate.

The key to our main results will be the following characterization of convexity. For completeness, we include a short proof of this folklore result; see Problem 12.Q of [4] for the equivalence of (i) and (iii) and also [5] for a long list of related results.

Proposition 2. For every continuous real-valued function $g$ on an interval $J$, the following assertions are equivalent:

(i) $g$ is convex on $J$;

(ii) for all $u, v \in J$, there exists some $t \in(0,1)$ for which

$$
g(t u+(1-t) v) \leq \operatorname{tg}(u)+(1-t) g(v)
$$

(iii) for all $u, v \in J$ with $u<v$, we have

$$
\frac{1}{v-u} \int_{u}^{v} g(x) \mathrm{d} x \leq \frac{g(u)+g(v)}{2} \text {. }
$$

Moreover, if $g$ is convex, then equality holds in the estimate of assertion (iii) precisely when $g$ is affine on $[u, v]$.

Proof. If $g$ is convex, then, for arbitrary distinct $u, v \in J$, the substitution rule yields

$$
\begin{aligned}
\frac{1}{v-u} \int_{u}^{v} g(x) \mathrm{d} x & =\int_{0}^{1} g(t u+(1-t) v) \mathrm{d} t \\
& \leq \int_{0}^{1}[\operatorname{tg}(u)+(1-t) g(v)] \mathrm{d} t \\
& =\frac{g(u)+g(v)}{2},
\end{aligned}
$$

which shows that (i) implies (iii). Next, if (ii) fails to hold, then there exist distinct points $u, v \in J$ for which $g(t u+(1-t) v)>$ $\operatorname{tg}(u)+(1-t) g(v)$ for all $t \in(0,1)$. Arguing as in the preceding step, integration over the interval $[0,1]$ then reveals that (iii) is violated; hence (iii) implies (ii). Finally, if $g$ is not convex, then there exist points $\alpha, \beta, \gamma \in J$ with $\alpha<\beta<\gamma$ such that $\ell(\beta)<g(\beta)$, where $\ell$ denotes the function representing the secant line from $(\alpha, g(\alpha))$ to $(\gamma, g(\gamma))$. For

$$
\begin{aligned}
& u=\sup \{x \in[\alpha, \beta]: g(x)=\ell(x)\}, \\
& v=\inf \{x \in[\beta, \gamma]: g(x)=\ell(x)\}
\end{aligned}
$$

we conclude from the continuity of $g$ that $\alpha \leq u<\beta<v \leq \gamma$, $g(u)=\ell(u), g(v)=\ell(v)$, and $\ell<g$ on $(u, v)$, which shows that condition (ii) cannot be satisfied. Thus (ii) implies (i), and consequently all the three assertions are indeed equivalent.

To establish the last claim, we have another look at the first step of our proof. Hence it is immediate that equality holds in (iii) if $g$ is affine on $[u, v]$. Conversely, suppose that $g$ is convex but not affine on $[u, v]$. By Lemma 1 , we obtain $g(t u+(1-$ $t) v)<\operatorname{tg}(u)+(1-t) g(v)$ for all $t \in(0,1)$. Another glance at the first step of the proof then confirms that strict inequality is obtained in (iii), as desired.

We conclude this section with the strict counterpart of the preceding result.

Corollary 3. For every continuous real-valued function $g$ on an interval $J$, the following assertions are equivalent: 
(i) $g$ is strictly convex on $J$;

(ii) for all distinct $u, v \in J$, there exists some $t \in(0,1)$ for which

$$
g(t u+(1-t) v)<\operatorname{tg}(u)+(1-t) g(v)
$$

(iii) for all $u, v \in J$ with $u<v$, we have

$$
\frac{1}{v-u} \int_{u}^{v} g(x) \mathrm{d} x<\frac{g(u)+g(v)}{2} .
$$

Proof. The implication (i) $\Longrightarrow$ (ii) is trivial, and (ii) $\Longrightarrow$ (iii) follows from Proposition 2, since (ii) ensures that there is no nontrivial subinterval of $J$ on which $g$ is affine. Finally, (iii) $\Longrightarrow$ (i) is immediate from Lemma 1 and Proposition 2.

\section{Main Sandwich Results}

Throughout this section, let $a$ and $b$ be real numbers with $a<b$, and let $f:[a, b] \longrightarrow \mathbb{R}$ be a continuously differentiable function. We first associate with this function two polynomials that will serve as upper and lower bounds in our sandwich results.

Lemma 4. There exist unique polynomials $p$ and $q$ of degree at most two for which

$$
\begin{aligned}
p(a) & =f(a), \\
p(b) & =f(b), \\
p^{\prime}(a) & =f^{\prime}(a), \\
q(a) & =f(a), \\
q(b) & =f(b), \\
q^{\prime}(b) & =f^{\prime}(b) ;
\end{aligned}
$$

namely, the polynomials $p(x)=\alpha+\beta x+\gamma x^{2}$ and $q(x)=$ $\widehat{\alpha}+\widehat{\beta} x+\widehat{\gamma} x^{2}$ with coefficients

$$
\begin{aligned}
\alpha= & \frac{1}{(b-a)^{2}}\left(\left(b^{2}-2 a b\right) f(a)+a^{2} f(b)\right. \\
& \left.-(b-a) a b f^{\prime}(a)\right) \\
\beta & =\frac{b+a}{b-a} f^{\prime}(a)-2 a \frac{f(b)-f(a)}{(b-a)^{2}} \\
\gamma= & \frac{f(b)-f(a)}{(b-a)^{2}}-\frac{f^{\prime}(a)}{b-a}, \\
\widehat{\alpha}= & \frac{1}{(b-a)^{2}}\left(b^{2} f(a)+\left(a^{2}-2 a b\right) f(b)\right. \\
& \left.+(b-a) a b f^{\prime}(b)\right)
\end{aligned}
$$

$$
\begin{aligned}
& \widehat{\beta}=-\frac{b+a}{b-a} f^{\prime}(b)+2 b \frac{f(b)-f(a)}{(b-a)^{2}} \\
& \widehat{\gamma}=-\frac{f(b)-f(a)}{(b-a)^{2}}+\frac{f^{\prime}(b)}{b-a}
\end{aligned}
$$

Proof. The result follows from the solution of two obvious linear systems, each of them consisting of three linear equations for three unknowns. We leave the elementary details to the reader.

Hereafter, we refer to the polynomials $p$ and $q$ from Lemma 4 as the polynomials associated with $f$.

Lemma 5. Suppose that $f^{\prime}$ is either strictly convex or strictly concave on $[a, b]$. Then the polynomials $p$ and $q$ associated with $f$ satisfy $p(x) \neq f(x)$ and $q(x) \neq f(x)$ for all $x \in(a, b)$.

Proof. First suppose that $f^{\prime}$ is strictly convex, and assume that $p(x)=f(x)$ for some $x \in(a, b)$. Then Rolle's theorem applied to the function $f-p$ on each of the intervals $[a, x]$ and $[x, b]$ ensures that there exist points $u$ and $v$ for which $a<u<x<$ $v<b$ such that $p^{\prime}(u)=f^{\prime}(u)$ and $p^{\prime}(v)=f^{\prime}(v)$. Because $p^{\prime}(a)=f^{\prime}(a)$, we conclude that $f^{\prime}-p^{\prime}$ vanishes at the three points $a, u$, and $v$. On the other hand, $p^{\prime}$ is affine, since the degree of $p$ is at most two. Thus $f^{\prime}-p^{\prime}$ inherits strict convexity from $f^{\prime}$. We conclude that $f^{\prime}(u)-p^{\prime}(u)<0$, the desired contradiction. Consequently, $p(x) \neq f(x)$ for all $x \in(a, b)$ when $f^{\prime}$ is strictly convex. The remaining three cases may be handled mutatis mutandis.

If the derivative of $f$ is either strictly convex or strictly concave on $[a, b]$, then a simple modification of the preceding argument shows that, for every quadratic polynomial $r$, the equation $r(x)=f(x)$ has at most three solutions $x \in[a, b]$. This result may be viewed as a general version of Théorème III on page 192 in Charbonnier's monograph [1]. As an immediate consequence, Charbonnier notes, without any further proof, that the trajectory traced by an arbitrary projectile is always sandwiched by certain canonical polynomials. We agree with Groetsch [2] that more work is needed for this conclusion. In fact, one of the main purposes of the theory of parabolic sandwiches is to bridge this gap. A complete proof of Charbonnier's result will be provided in Corollary 13 below.

It turns out that the theory of parabolic sandwiches is dominated by the relationship between the quantities

$$
\begin{aligned}
& \frac{f^{\prime}(a)+f^{\prime}(b)}{2}, \\
& \frac{f(b)-f(a)}{b-a} .
\end{aligned}
$$

To simplify notation, we introduce

$$
\diamond(f, a, b)=\frac{f^{\prime}(a)+f^{\prime}(b)}{2}-\frac{f(b)-f(a)}{b-a} .
$$

The inequalities $\diamond(f, a, b) \leq 0$ and $\diamond(f, a, b) \geq 0$ admit simple geometric interpretations in terms of certain tangent lines, as shown next. 
Lemma 6. Suppose that $f^{\prime}(a) \neq f^{\prime}(b)$, and let $c$ denote the $x$-coordinate of the point of intersection of the tangent lines for $f$ through the points $(a, f(a))$ and $(b, f(b))$. If $f^{\prime}(a)>f^{\prime}(b)$, then $\diamond(f, a, b) \leq 0$ precisely when $c \geq(a+b) / 2$, while if $f^{\prime}(a)<$ $f^{\prime}(b)$, then $\diamond(f, a, b) \leq 0$ precisely when $c \leq(a+b) / 2$.

Proof. The two tangent lines are given by $\ell_{a}(x)=f(a)+$ $f^{\prime}(a)(x-a)$ and $\ell_{b}(x)=f(b)+f^{\prime}(b)((x-b)$ for all $x \in \mathbb{R}$, and it is easily seen that the identity $\ell_{a}(c)=\ell_{b}(c)$ holds exactly when

$$
c=\frac{f(b)-f(a)+a f^{\prime}(a)-b f^{\prime}(b)}{f^{\prime}(a)-f^{\prime}(b)} .
$$

The verification of the stipulated equivalences is then straightforward.

The following two auxiliary results collect the information about the sign of $\diamond(f, a, b)$ which is needed in the proof of our main results.

Lemma 7. In the setting of Lemma 4, we have

$$
\begin{aligned}
& f^{\prime}(a) \leq q^{\prime}(a) \Longleftrightarrow \\
& f^{\prime}(b) \leq p^{\prime}(b) \Longleftrightarrow \\
& \diamond(f, a, b) \leq 0 .
\end{aligned}
$$

Moreover, the same equivalences hold when " $\leq$ " is replaced throughout by either " $\geq$ " or "=".

Proof. With the notation of Lemma 4, the estimate $f^{\prime}(a) \leq$ $q^{\prime}(a)$ holds precisely when $f^{\prime}(a) \leq \widehat{\beta}+2 \widehat{\gamma} a$, which may be rewritten in the form

$$
f^{\prime}(a) \leq 2 \frac{f(b)-f(a)}{b-a}-f^{\prime}(b)
$$

and hence is equivalent to $\diamond(f, a, b) \leq 0$. Similar arguments show that $\diamond(f, a, b) \leq 0$ holds exactly when $f^{\prime}(b) \leq p^{\prime}(b)$ and that the equivalences remain valid for the case of " $\geq$ " instead of " $\leq$ ". The remaining assertion regarding " $=$ " is then immediate.

Lemma 8. The polynomials $p$ and $q$ associated with a function $f$ satisfy the following:

(a) the identity $\diamond(f, a, b)=0$ holds precisely when $p=q$;

(b) $p \leq f \leq q$ on $[a, b]$ implies that $\diamond(f, a, b) \leq 0$;

(c) $q \leq f \leq p$ on $[a, b]$ implies that $\diamond(f, a, b) \geq 0$;

(d) if $f^{\prime}$ is convex, then $\diamond(f, a, b) \geq 0$, and $\diamond(f, a, b)>0$ unless $f^{\prime}$ is affine on $[a, b]$;

(e) if $f^{\prime}$ is concave, then $\diamond(f, a, b) \leq 0$, and $\diamond(f, a, b)<0$ unless $f^{\prime}$ is affine on $[a, b]$.

Proof. (a) The equality version of Lemma 7 confirms that the identity $\diamond(f, a, b)=0$ holds exactly when $f^{\prime}(a)=q^{\prime}(a)$. By the uniqueness assertion of Lemma 4, this means precisely that $p=q$. obtain

(b) From $p \leq f \leq q$ on $[a, b]$ and $p(a)=f(a)=q(a)$, we

$$
\frac{p(x)-p(a)}{x-a} \leq \frac{q(x)-q(a)}{x-a}
$$

for all $x \in(a, b)$. Taking the limit as $x \longrightarrow a^{+}$then yields $p^{\prime}(a) \leq q^{\prime}(a)$ and hence $f^{\prime}(a) \leq q^{\prime}(a)$. Lemma 7 now completes the argument.

(c) Since the polynomials associated with $-f$ in the sense of Lemma 4 are $-p$ and $-q$, the estimate $-p \leq-f \leq-q$ on $[a, b]$ entails, by part (b), that $-\diamond(f, a, b)=\diamond(-f, a, b) \leq 0$ and hence the assertion. Of course, one could also proceed as in the proof of (b).

(d) This follows from an application of Proposition 2 to $g=f^{\prime}$ and $(u, v)=(a, b)$ in assertion (iii).

(e) This final claim is immediate from part (d) applied to $-f$.

We mention in passing that, for every differentiable function $f$ on $[a, b]$, the convexity or concavity of $f^{\prime}$ ensures that $f^{\prime}$ is automatically continuous on the entire closed interval $[a, b]$, not just on its interior. Indeed, it is a wellknown consequence of the convexity or concavity condition that the one-sided limits of $f^{\prime}$ exist at the endpoints $a$ and $b$, and it follows from the classical Darboux theorem that these one-sided limits coincide with $f^{\prime}(a)$ and $f^{\prime}(b)$, respectively; see Lemma 4.48 of [6] and Theorem 5.12 of [7].

Theorem 9. A function $f$ is sandwiched by its associated polynomials $p$ and $q$ as follows:

(a) if $f^{\prime}$ is strictly concave, then $p<f<q$ on $(a, b)$;

(b) if $f^{\prime}$ is concave, then $p \leq f \leq q$ on $[a, b]$;

(c) if $f^{\prime}$ is strictly convex, then $q<f<p$ on $(a, b)$;

(d) if $f^{\prime}$ is convex, then $q \leq f \leq p$ on $[a, b]$.

Proof. (a) By part (e) of Lemma 8, the strict concavity of $f^{\prime}$ implies that $\diamond(f, a, b)<0$. Hence we conclude from Lemma 7 that $f^{\prime}(a)<q^{\prime}(a)$ and $f^{\prime}(b)<p^{\prime}(b)$. The first of these inequalities ensures that

$$
\frac{f(x)-f(a)}{x-a}<\frac{q(x)-q(a)}{x-a}
$$

for all $x \in(a, b)$ sufficiently close to $a$ and hence $f(x)<q(x)$ for all such $x$, because $f(a)=q(a)$. On the other hand, we know from Lemma 5 that $f(x) \neq q(x)$ for all $x \in(a, b)$. Consequently, by the intermediate value theorem, $f(x)<$ $q(x)$ for arbitrary $x \in(a, b)$. Similar reasoning shows that the inequality $f^{\prime}(b)<p^{\prime}(b)$ yields $p(x)<f(x)$ for all $x \in(a, b)$.

(b) To reduce this claim to the preceding one, we consider an arbitrary $\varepsilon>0$ and define $f_{\varepsilon}(x)=f(x)-\varepsilon x^{3}$ for all $x \in$ $[a, b]$. Since $f_{\varepsilon}^{\prime}(x)=f^{\prime}(x)-3 \varepsilon x^{2}$ for all $x \in[a, b]$, it follows that $f_{\varepsilon}^{\prime}$ is strictly concave on $[a, b]$ as the sum of a concave and a strictly concave function. Consequently, if $p_{\varepsilon}$ and $q_{\varepsilon}$ denote the polynomials associated with $f_{\varepsilon}$, then assertion (a) 
ensures that $p_{\varepsilon}(x)<f_{\varepsilon}(x)<q_{\varepsilon}(x)$ for all $x \in(a, b)$. A glance at the formulas for these polynomials in Lemma 4 reveals that $p_{\varepsilon}(x) \longrightarrow p(x)$ and $q_{\varepsilon}(x) \longrightarrow q(x)$ as $\varepsilon \longrightarrow 0^{+}$for each $x \in$ $[a, b]$. Thus $p(x) \leq f(x) \leq q(x)$ for all $x \in[a, b]$, as desired.

((c) and (d)) These assertions follow from parts (a) and (b), respectively, applied to $-f$, since, as already noted in the proof of part (c) of Lemma 8, the polynomials associated with $-f$ are $-p$ and $-q$.

Corollary 10. If $p$ and $q$ are the polynomials associated with a cubic polynomial $f$, then $p<f<q$ on $(a, b)$ in the case $f^{\prime \prime \prime}<0$, while $q<f<p$ on $(a, b)$ in the case $f^{\prime \prime \prime}>0$.

Proof. The result is immediate from Theorem 9, since the quadratic polynomial $f^{\prime}$ is strictly concave in the case $f^{\prime \prime \prime}<0$ and strictly convex otherwise.

It is fairly easy to construct examples of functions that are sandwiched by their associated polynomials in one way or the other but fail to be convex or concave. In fact, such examples may be found among small perturbations of strictly convex or strictly concave functions. To obtain a certain converse of Theorem 9, we need localized versions of our sandwich properties. For arbitrary $u, v \in[a, b]$ with $u<v$, let $p_{u, v}$ and $q_{u, v}$ denote the polynomials associated with the function $f$ when restricted to $[u, v]$.

Theorem 11. For every continuously differentiable function $f$ on $[a, b]$, the following equivalences hold:

(a) $f^{\prime}$ is concave on $[a, b]$ precisely when $p_{u, v} \leq f \leq q_{u, v}$ on $[u, v]$ for all $u, v \in[a, b]$ with $u<v$;

(b) $f^{\prime}$ is strictly concave on $[a, b]$ precisely when $p_{u, v}<f<$ $q_{u, v}$ on $(u, v)$ for all $u, v \in[a, b]$ with $u<v$;

(c) $f^{\prime}$ is convex on $[a, b]$ precisely when $q_{u, v} \leq f \leq p_{u, v}$ on $[u, v]$ for all $u, v \in[a, b]$ with $u<v$;

(d) $f^{\prime}$ is strictly convex on $[a, b]$ precisely when $q_{u, v}<f<$ $p_{u, v}$ on $(u, v)$ for all $u, v \in[a, b]$ with $u<v$.

Proof. One implication of assertion (c) is, of course, immediate from part (d) of Theorem 9, while the other one follows from part (c) of Lemma 8 together with Proposition 2 applied to $g=f^{\prime}$. This also settles assertion (a), since by now it should be obvious that (a) and (c) are equivalent. Similarly, assertions (b) and (d) follow from Corollary 3, Lemma 8, and Theorem 9.

\section{Application to Projectile Motion}

We first describe a general setting for the motion of a projectile in the $x y$-plane. Throughout we consider a projectile that is launched at time $t=0$ from the origin with muzzle speed $s>0$ and angle of inclination $\theta \in(-\pi / 2, \pi / 2)$ relative to the positive $x$-axis. The position vector of the projectile at time $t \geq 0$ is denoted by $\mathbf{r}(t)=\langle x(t), y(t)\rangle$.

We suppose that the motion of the projectile is governed by two forces. As usual, one of these forces is gravity in the direction of the negative $y$-axis, which results in acceleration of a given magnitude $g>0$. The other force is air resistance whose direction is opposite to the velocity vector $\mathbf{r}^{\prime}(t)=\left\langle x^{\prime}(t), y^{\prime}(t)\right\rangle$ of the projectile and whose magnitude is proportional to the mass $m$ of the projectile but otherwise quite arbitrary. Specifically, the retarding force is supposed to be represented by a vector of the form

$$
-m W\left(t, x(t), x^{\prime}(t), y(t), y^{\prime}(t)\right)\left\langle x^{\prime}(t), y^{\prime}(t)\right\rangle,
$$

where $W$ is a given nonnegative continuous function of five variables, defined on a suitable domain in $\mathbb{R}^{5}$. In addition to the classical case of no air resistance represented by $W=$ 0 , this model covers the important case where the drag is proportional to some power of the speed

$$
\left|\mathbf{r}^{\prime}(t)\right|=\sqrt{x^{\prime}(t)^{2}+y^{\prime}(t)^{2}}
$$

of the projectile; see Chapter 3 of [8]. It also allows, for instance, for drag depending on the altitude $y(t)$ of the projectile.

By Newton's law, the motion of the projectile is then described by the initial value problem

$$
\begin{aligned}
& x^{\prime \prime}(t)=-W\left(t, x(t), x^{\prime}(t), y(t), y^{\prime}(t)\right) x^{\prime}(t), \\
& y^{\prime \prime}(t)=-g-W\left(t, x(t), x^{\prime}(t), y(t), y^{\prime}(t)\right) y^{\prime}(t), \\
& x(0)=0, \\
& y(0)=0, \\
& x^{\prime}(0)=s \cos (\theta), \\
& y^{\prime}(0)=s \sin (\theta) .
\end{aligned}
$$

In the following, we assume that the drag function $W$ is admissible in the sense that, for every choice of $s>0$ and $-\pi / 2<\theta<\pi / 2$, the initial value problem $(\triangle)$ has a unique solution $(x(t), y(t))$ that is defined for all $t \geq 0$. In practice, admissibility is guaranteed by existence and uniqueness results of Picard-Lindelöf type under mild Lipschitz conditions on the function $W$ with respect to the last four variables. In particular, $W$ is admissible if the partial derivatives of $W$ with respect to each of the last four variables exist and are continuous; see, for instance, [9].

At the present level of generality, explicit formulas for the solutions $x(t)$ and $y(t)$ of $(\triangle)$ are clearly out of reach. In fact, even in the special case of drag quadratic in speed, no such formulas are known; see, for example, $[8,10]$. Nevertheless, we will be able to obtain remarkable geometric insight into the shape of the trajectory traced by the projectile.

The clue is to focus directly on $(\triangle)$ and the well-defined continuous function $\omega$ given by

$$
\omega(t)=W\left(t, x(t), x^{\prime}(t), y(t), y^{\prime}(t)\right)
$$

for all $t \geq 0$. Even though, in general, no explicit formula for $\omega$ is available, standard separation of variables shows that the solution of the differential equation $z^{\prime}(t)=-\omega(t) z(t)$ is given by

$$
z(t)=c \exp \left(-\int_{0}^{t} \omega(\tau) \mathrm{d} \tau\right)
$$


for all $t \geq 0$, where $c$ is a real constant. Because $c=z(0)$, we conclude from $(\triangle)$ that

$$
x^{\prime}(t)=z(t)=s \cos (\theta) \exp \left(-\int_{0}^{t} \omega(\tau) \mathrm{d} \tau\right)
$$

and therefore $x^{\prime}(t)>0$ for all $t \geq 0$. It follows that the function $x$ is strictly increasing on $[0, \infty)$ and that its range is an interval of the form $\left[0, x_{\infty}\right)$ where $0<x_{\infty} \leq \infty$. For instance, it is well known that $x_{\infty}=\infty$ in the classical case of no drag, while $x_{\infty}<\infty$ in the case of drag that is either linear or quadratic in speed; see again $[8,10]$. Moreover, the inverse of the function $x$ allows us to express the flight curve of our projectile in terms of a function $y=f(x)$ on $\left[0, x_{\infty}\right)$ which will be investigated in the following result.

Theorem 12. Let $W$ denote an admissible drag function, let $(x(t), y(t))$ for $t \geq 0$ denote the solution of the corresponding initial value problem $(\triangle)$, and consider the composition $f=$ $y \circ x^{-1}$ on the range $\left[0, x_{\infty}\right)$ of $x$. Then $f$ satisfies

$$
f(x(t))=y(t)
$$

for all $t \geq 0$, and its third derivative exists and is continuous on $\left[0, x_{\infty}\right)$. Moreover, $f$ is strictly concave, while $f^{\prime}$ is always concave and even strictly concave provided that $W>0$.

Proof. We first observe that $x^{-1}$ inherits differentiability from $x$, since we know that $x^{\prime}(t)>0$ for all $t \geq 0$. From the definition of $f$, it is then immediate that $f$ is differentiable and satisfies the equation $f(x(t))=y(t)$ for all $t \geq 0$. Hence the chain rule implies that $f^{\prime}(x(t)) x^{\prime}(t)=y^{\prime}(t)$ and therefore

$$
f^{\prime}(x(t))=\frac{y^{\prime}(t)}{x^{\prime}(t)}
$$

for all $t \geq 0$. Since $(\triangle)$ confirms that both $x^{\prime}$ and $y^{\prime}$ are differentiable, so is $f^{\prime}$. Moreover, taking the derivative of the preceding identity and using $(\triangle)$, we obtain

$$
\begin{aligned}
f^{\prime \prime} & (x(t)) x^{\prime}(t)=\frac{x^{\prime}(t) y^{\prime \prime}(t)-x^{\prime \prime}(t) y^{\prime}(t)}{x^{\prime}(t)^{2}} \\
& =\frac{-x^{\prime}(t)\left[g+\omega(t) y^{\prime}(t)\right]+\omega(t) x^{\prime}(t) y^{\prime}(t)}{x^{\prime}(t)^{2}} \\
& =\frac{-g}{x^{\prime}(t)}
\end{aligned}
$$

and consequently

$$
f^{\prime \prime}(x(t))=-\frac{g}{x^{\prime}(t)^{2}}<0
$$

for all $t \geq 0$. In particular, this shows that $f$ is strictly concave on $\left[0, x_{0}\right)$. Repeating the procedure, we arrive at

$$
f^{\prime \prime \prime}(x(t)) x^{\prime}(t)=2 g x^{\prime}(t)^{-3} x^{\prime \prime}(t)
$$

and therefore

$$
f^{\prime \prime \prime}(x(t))=\frac{2 g x^{\prime \prime}(t)}{x^{\prime}(t)^{4}}=-\frac{2 g \omega(t)}{x^{\prime}(t)^{3}} \leq 0
$$

for all $t \geq 0$, again on account of $(\triangle)$. We conclude that $f^{\prime \prime \prime}$ is continuous and that $f^{\prime}$ is always concave. Of course, $f^{\prime}$ is actually affine in the classical case of no air resistance $W=0$, while the condition $W>0$ ensures that $f^{\prime \prime \prime}<0$ and hence that $f^{\prime}$ is strictly concave, as claimed.

As a simple application of the preceding results, we now obtain an extended version of Charbonnier's Corollary 3; see page 193 of [1].

Corollary 13. In the setting of Theorem 12, consider an arbitrary time $\tau>0$, and define the polynomials

$$
\begin{aligned}
p_{\tau}(z)= & \tan (\theta) z+\left(\frac{y(\tau)}{x(\tau)^{2}}-\frac{\tan (\theta)}{x(\tau)}\right) z^{2} \\
q_{\tau}(z)= & \left(2 \frac{y(\tau)}{x(\tau)}-\frac{y^{\prime}(\tau)}{x^{\prime}(\tau)}\right) z \\
& +\left(\frac{y^{\prime}(\tau)}{x(\tau) x^{\prime}(\tau)}-\frac{y(\tau)}{x(\tau)^{2}}\right) z^{2}
\end{aligned}
$$

Then the function $f=y \circ x^{-1}$ induced by the solution curve $(x(t), y(t))$ of the initial value problem $(\triangle)$ is sandwiched by $p_{\tau}$ and $q_{\tau}$ in the sense that

$$
p_{\tau} \leq f \leq q_{\tau}
$$

on the interval $[0, x(\tau)]$. Moreover, strict inequalities hold on $(0, x(\tau))$ provided that $W>0$.

Proof. The result is immediate from Theorems 9 and 12 together with Lemma 4 for the choices $a=0$ and $b=$ $x(\tau)$.

We conclude with a typical numerical example. Let $g=$ $9.81 \mathrm{~m} / \mathrm{sec}^{2}, s=100 \mathrm{~m} / \mathrm{sec}$, and $\theta=\pi / 6$ radian, and consider the case of air resistance which is proportional to the square of the speed. This classical case is addressed by the initial value problem

$$
\begin{gathered}
x^{\prime \prime}(t)=-\gamma \sqrt{x^{\prime}(t)^{2}+y^{\prime}(t)^{2}} x^{\prime}(t), \\
x^{\prime}(0)=s \cos (\theta), x(0)=0, \\
y^{\prime \prime}(t)=-g-\gamma \sqrt{x^{\prime}(t)^{2}+y^{\prime}(t)^{2}} y^{\prime}(t), \\
y^{\prime}(0)=s \sin (\theta), y(0)=0,
\end{gathered}
$$

where we choose the quadratic air resistance coefficient to be $\gamma=0.0025 \mathrm{~m}^{-1}$; see [8].

Although no explicit solution formula is known, one may use the NDSolve command of Mathematica to obtain a numerical solution based on some Runge-Kutta type method. The graphics in Figure 2 show the corresponding trajectory of the projectile over the time period of 10 seconds together with two pairs of (dashed) sandwiching parabolas for the choices $\tau=7 \mathrm{sec}$ and $\tau=10 \mathrm{sec}$.

We note that the case of projectile motion is rather special, since here both the function and its derivative happen to be 


\section{ROBERT KANTROWITZ AND MICHAEL M. NEUMANN}

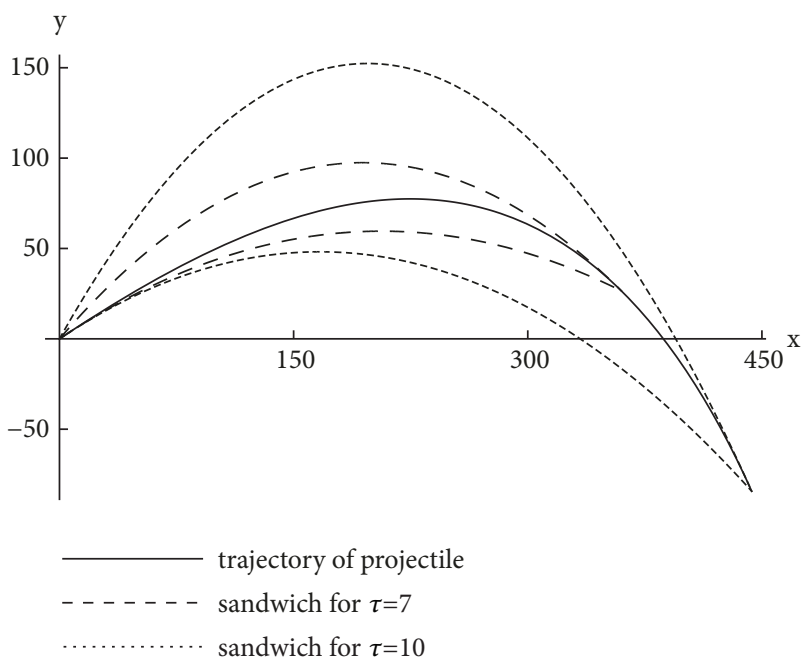

FIGURE 2

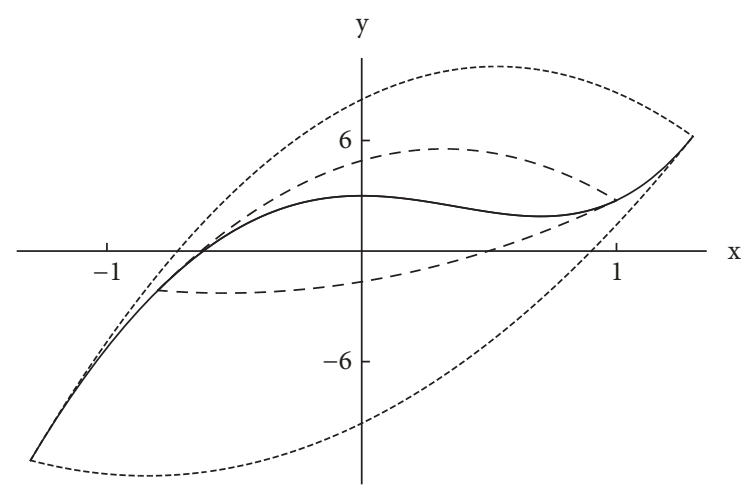

FIGURE 3

\section{References}

[1] P. Charbonnier, Balistique Extérieure Rationnelle, Doin, Paris, 1907.

[2] C. W. Groetsch, “The commandant's corollary," American Mathematical Monthly, vol. 107, pp. 15-23, 2000.

[3] G. H. Hardy, J. E. Littlewood, and G. Pólya, Inequalities, Cambridge University Press, Cambridge, UK, 1934.

[4] A. W. Roberts and D. E. Varberg, Convex Functions, Academic Press, New York, NY, USA, 1973.

[5] Y.-C. Li and C.-C. Yeh, "Some characterizations of convex functions," Computers and Mathematics with Applications, vol. 59, pp. 327-337, 2010.

[6] K. R. Stromberg, Introduction to Classical Real Analysis, Wadsworth, Belmont, 1981.

[7] W. Rudin, Principles of Mathematical Analysis, McGraw-Hill, New York, NY, USA, 3rd edition, 1976.

[8] N. de Mestre, The Mathematics of Projectiles in Sport, Cambridge University Press, Cambridge, UK, 1990.

[9] E. A. Coddington and N. Levinson, Theory of Ordinary Differential Equations, McGraw-Hill, New York, NY, USA, 1955.

[10] W. W. Hackborn, "Motion through air: what a drag," Canadian Applied Mathematics Quarterly, vol. 14, pp. 285-298, 2006.

concave. However, Theorem 11 holds in a more general setting and leads, as indicated earlier, to a geometric visualization of the conditions $f^{\prime \prime \prime} \geq 0$ or $f^{\prime \prime \prime} \leq 0$ on a given interval. Indeed, if the third derivative of a function $f$ exists on an interval $[a, b]$, then the condition $f^{\prime \prime \prime} \geq 0$ on $[a, b]$ is equivalent to the sandwich estimate $q_{u, v} \leq f \leq p_{u, v}$ on $[u, v]$ for arbitrary points $u, v \in[a, b]$ with $u<v$. We illustrate this characterization by the example of the function $f(x)=$ $3 \cos (2 x)+4 x^{3}$ which satisfies $f^{\prime \prime \prime}(x)=24 \sin (2 x)+24 \geq 0$ for all $x \in \mathbb{R}$. The graphics in Figure 3 display this function on the interval $[-1.3,1.3]$ together with two typical parabolic sandwiches.

\section{Data Availability}

No data were used to support this study.

\section{Conflicts of Interest}

The authors declare that they have no conflicts of interest. 


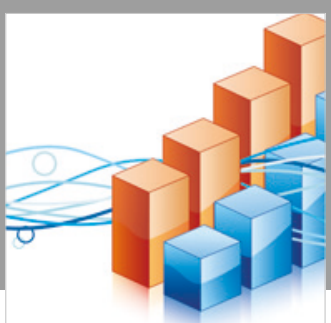

Advances in

Operations Research

\section{-n-m}
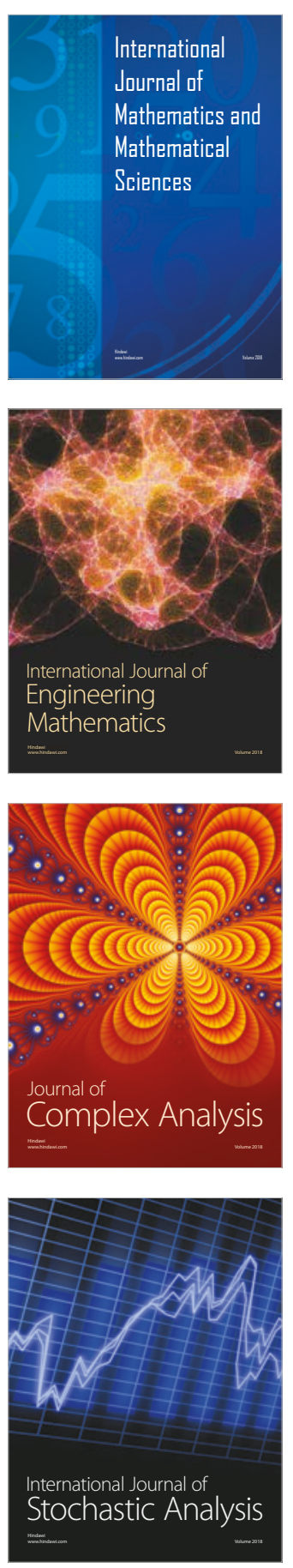
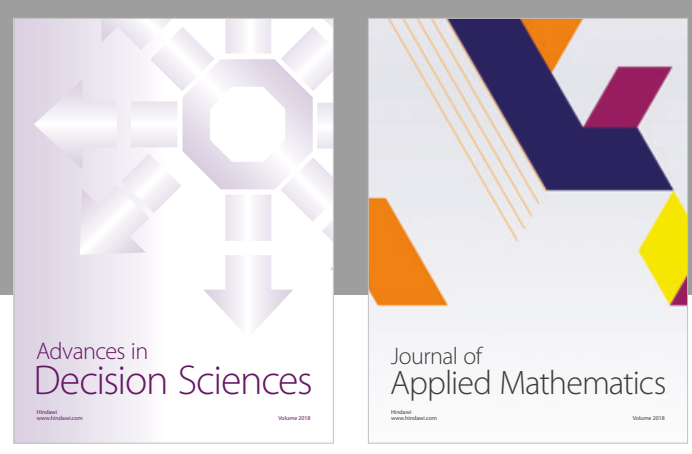

Journal of

Applied Mathematics
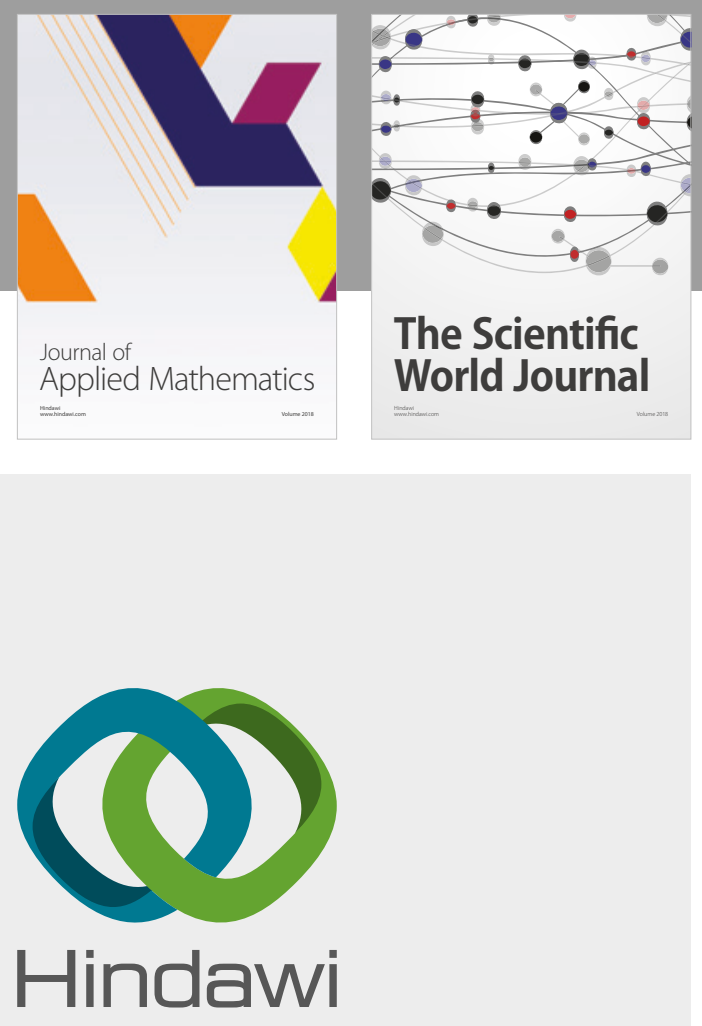

Submit your manuscripts at

www.hindawi.com

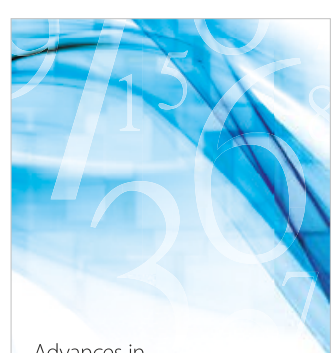

Advances in
Numerical Analysis
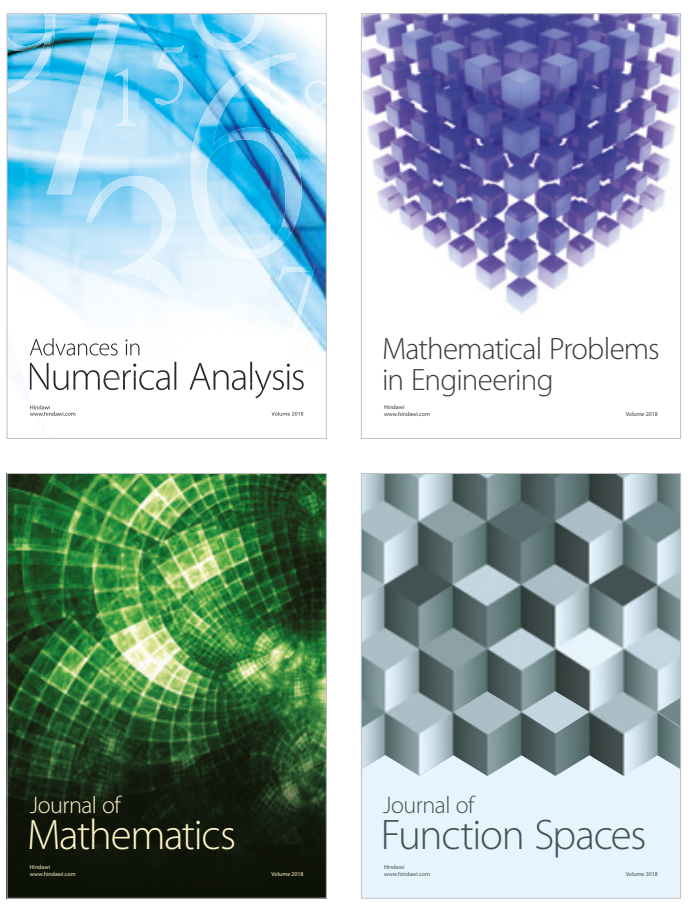

Mathematical Problems in Engineering

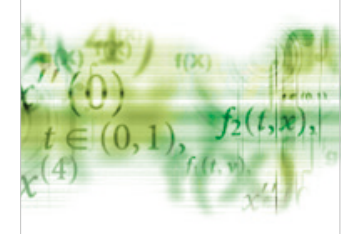

International Journal of

Differential Equations

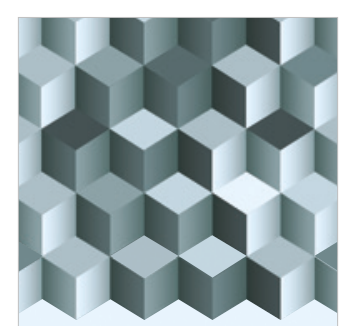

Journal of

Function Spaces

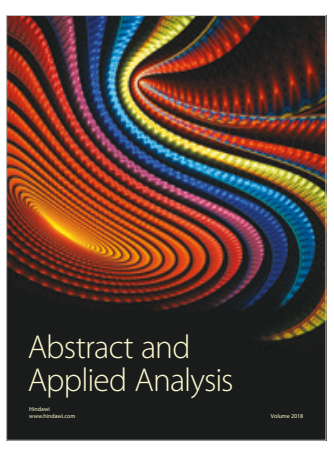

The Scientific

World Journal

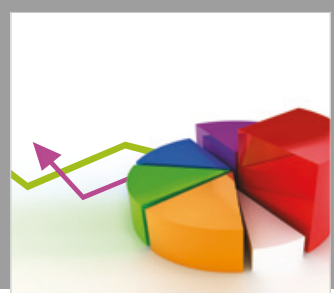

Journal of

Probability and Statistics
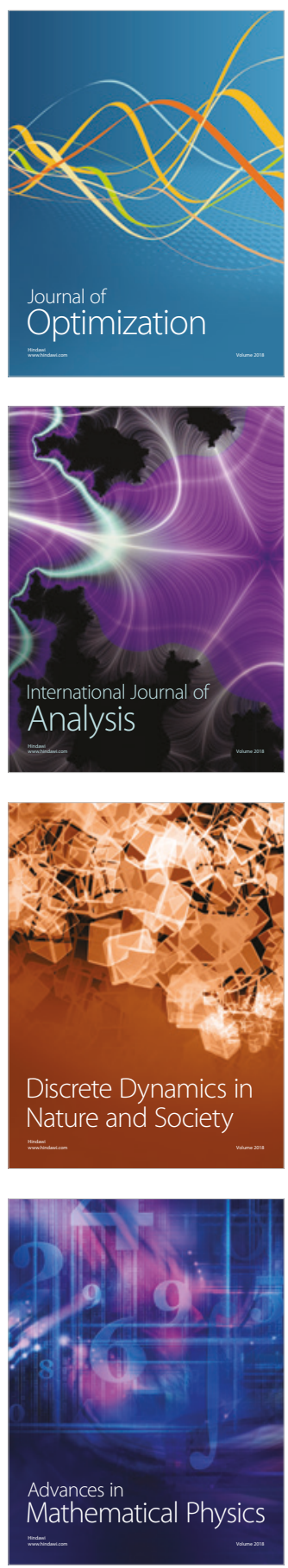